\title{
Prostate Cancer in Black Men: Is It Time for Personalized Screening Approaches?
}

\author{
Lauren P. Wallner, PhD, MPH (iD) ${ }^{1,2,3}$ and Steven J. Jacobsen, MD, PhD 3
}

Is prostate cancer different in black men? This is the question that Tsodikov and colleagues are trying to answer in their study published in this issue of Cancer. ${ }^{1}$ Their findings highlight some important racial differences in screening patterns and projected outcomes that suggest a more personalized approach to prostate cancer screening that is not "1 size fits all." Indeed, a more personalized approach to screening among black men would be an active move toward precision medicine that does not require the sequencing of germline or somatic DNA.

To inform this question, the authors used predictive modeling techniques to compare the natural history of prostate cancer among black men compared with the general population. To do this, they reconstructed prostate-specific antigen (PSA) screening patterns in the United States and then fit 3 independent simulation models using screening data from the National Health Interview Survey and prostate cancer incidence data from the Surveillance, Epidemiology, and End Results (SEER) database. They observed that from 30\% to $43 \%$ of black men develop preclinical prostate cancer by age 85 years, a risk that is $28 \%$ to $56 \%$ higher than that in the general population. The models predicted similar risks of prostate cancer diagnosis when comparing black men with men in the general population (range, 35\%-49\%). Black men, however, were much more likely (range, 44\%-75\%) to progress to metastatic prostate cancer before diagnosis compared with men in the general population.

Their study is timely, as the US Preventive Services Task Force (USPSTF) is currently reviewing their 2012 grade D recommendation against PSA screening ${ }^{2}$ and is expected to release an updated recommendation sometime in 2017. One of the key issues highlighted in the research plan released as part of the review is evaluating whether the effectiveness of PSA screening varies by subpopulation or risk factor, including age, race/ethnicity, family history, and clinical risk assessment. ${ }^{3}$ The 2012 USPSTF prostate cancer screening recommendations do not currently take into account race/ethnicity; the grade D recommendation against screening applies to men of all races. ${ }^{2}$ The appropriateness of these recommendations for higher-risk men, including black men, continues to be debated in the medical literature and in the media. ${ }^{4-6}$ In addition, the clinical guidelines in this area are not aligned when it comes to screening black men. Although the American Urological Association and National Comprehensive Cancer Network guidelines both reference that African American race is a strong risk factor for prostate cancer and that screening discussions should be individualized, ${ }^{7,8}$ the guidelines fall short of providing explicit guidance on incorporating race/ethnicity into screening recommendations and discussions. The American Cancer Society guidelines are the only ones that currently make specific screening recommendations for black men. They recommend offering PSA screening to black men beginning at age 45 years. ${ }^{9}$ Therefore, a lack of clarity continues around the appropriateness of using PSA to screen for prostate cancer in black men.

What is clear, however, is that prostate cancer disproportionally affects black men. In 2016 alone, 1 in 6 black men were diagnosed with prostate cancer, and 1 in 23 died from their disease. ${ }^{10}$ Black men are more likely to be diagnosed with prostate cancer at a younger age and a higher stage and to have their disease progress after treatment compared with white men. ${ }^{10}$ Although the incidence and mortality rates of prostate cancer in the United States have been steadily decreasing over time, black men continue to remain significantly more likely to die from their prostate cancer. ${ }^{10}$ The lifetime probability of a black man dying of prostate cancer is almost double that of a non-Hispanic white man (4.4\% vs $2.4 \%) .{ }^{10}$

This is not new news. African American race is 1 of the strongest and most established risk factors for prostate cancer diagnosis and death. A wealth of literature exists in this area and has spawned myriad studies aimed at elucidating the

\footnotetext{
Corresponding author: Lauren P. Wallner, PhD, MPH, Departments of Medicine and Epidemiology, University of Michigan, North Campus Research Complex, 2800 Plymouth Road, Building 16, 409E, Ann Arbor, MI 48109; Fax: (734) 232-0788; Iwallner@med.umich.edu

${ }^{1}$ Department of Medicine, University of Michigan, Ann Arbor, Michigan; ${ }^{2}$ Department of Epidemiology, University of Michigan, Ann Arbor, Michigan; ${ }^{3}$ Department of Research and Evaluation, Kaiser Permanente Southern California, Pasadena, California.

See referenced original article on pages 2312-9, this issue.
}

DOI: 10.1002/cncr.30685, Received: February 7, 2017; Accepted: February 18, 2017, Published online April 24, 2017 in Wiley Online Library (wileyonlinelibrary. com) 
underlying causes of these disparities, including sociodemographics, genetics, environmental factors, health behaviors, differences in tumor biology, access to care issues, and variations in screening, detection, treatment, and post-treatment surveillance. ${ }^{6,11}$ And yet there remains a lack of data on the effectiveness of prostate cancer screening in black men. It is well established that black men are under-represented in clinical trials of prostate cancer. ${ }^{12}$ In the 2 large, randomized controlled trials of prostate cancer screening on which the USPSTF based their recommendations, ${ }^{13,14}$ the proportion of black men included was extremely low. In the Prostate, Lung, Colorectal, and Ovarian Cancer Screening Trial, only $4 \%$ of the study population was African American. Although the European Randomized Study of Screening for Prostate Cancer did not publish their race data, the countries included were mostly Caucasian. ${ }^{6,14}$ Given the lack of prospective screening data in black men, additional observational studies that include more diverse populations are necessary to inform future screening strategies.

In addition, further consideration of more tailored prostate cancer screening guidelines is urgently needed. Recently, multiple studies suggest that the 2012 USPSTF guidelines have resulted in a marked decrease in PSA screening rates in the United States. These decreases in screening rates have been observed among all age ranges. ${ }^{15-17}$ In addition, studies have noted decreases in the rates of diagnosis of early stage disease. ${ }^{16,18}$ Disturbingly, early data suggest there may be an increase in highrisk disease, ${ }^{19}$ but use associated with PSA, including subsequent referrals, urology visits, and treatments, may not have changed much. ${ }^{17,20}$ Although it is possible that this may be because of the more discriminate use of PSA screening among men who physicians believe are most likely to benefit from early detection, it is concerning that the current recommendations may result in decreased screening and diagnosis rates in higher-risk men who are most likely to benefit from early detection and to require subsequent treatment.

It is noteworthy that the impact of these recommendations on metastatic and mortality rates still remains largely unknown. Using predictive modeling approaches, Gulati and colleagues previously estimated that, by eliminating PSA screening, the number of patients with metastatic disease would double, and the mortality rate would increase from $13 \%$ to $25 \%$ by $2025 .{ }^{21}$ Two recent studies suggest that the incidence of later stage or metastatic disease may be increasing since the release of the recommendations, particularly in younger men. ${ }^{22,23}$ However, because prostate cancer takes years to progress in most men, it is still too soon to fully appreciate the impact of the guidelines on the rates of metastatic prostate cancer and prostate cancer death. If the wide deimplementation of PSA screening does turn out to increase metastatic and fatal prostate cancer rates in the United States, then it is likely that this burden will disproportionately affect black men. Therefore, data to inform policy discussions regarding whether or not PSA screening guidelines should differ for black men, as presented in this issue of Cancer, are important.

The results reported by Tsodikov and colleagues further inform the ongoing discussion about whether or not screening practices in black men need to be adapted to better align with the finding that they are much more likely to progress to metastatic disease and subsequently die from their cancer. ${ }^{1}$ In light of the findings from their study, the authors question whether or not it is time to consider alternative screening approaches specifically in black men. However, there are some potential limitations to their study that warrant consideration when interpreting the results. The use of predictive modeling has inherent limitations, including the reliance on the assumption, in this case, that prostate cancer is progressive. This resulted in the exclusion from these models of indolent prostate cancers, which make up a large proportion of the prostate cancers diagnosed as a result of PSA screening. However, the authors did use 3 different modeling strategies that relied on various assumptions in an effort to increase the robustness of their results. In addition, the data used to reconstruct PSA screening were retrospective, and age at first PSA was self-reported from the 2005 National Health Interview Survey, which was then combined with claims from SEER-Medicare to estimate the intervals between screening tests. The older patients in SEER-Medicare may also have had screening intervals that differ from those in younger men, which is relevant, because the authors' recommendations about the policy implications of their work focus on screening black men at younger ages.

At the end of the day, however, the findings from this study suggest that the risk/benefit tradeoffs of PSA screening may be quite different for black men compared with men in the general population. Therefore, whether current general population PSA screening guidelines should apply to this high-risk group warrants further policy consideration. Given the issues of overdiagnosis and treatment of indolent prostate cancer that arose from prior ubiquitous screening practices, any recommendations to change current screening approaches need to be carefully considered and based on empirical evidence. Although 
more evidence on the downstream mortality effects of PSA screening in black men would be ideal to inform these policies, it is worth contemplating whether we have now reached a critical tipping point in PSA screening for black men. In the growing era of precision medicine and personalized health initiatives, it is important that we consider that precision medicine approaches can be applied more broadly than the use genetic or genomic data. These approaches can be applied to better tailor screening practices to men who will indeed benefit from early detection of prostate cancer. Because the evidence is accumulating that a 1-size-fits-all screening approach to prostate cancer may not be what is most appropriate, it may be time for the conversation around PSA screening to really focus on more personalized approaches to screening in high-risk black men.

\section{FUNDING SUPPORT}

No specific funding was disclosed.

\section{CONFLICT OF INTEREST DISCLOSURES}

Lauren P. Wallner reports a prior grant from GlaxoSmithKline outside the submitted work. Steven J. Jacobsen made no disclosures.

\section{REFERENCES}

1. Tsodikov A, Gulati R, de Carvalho TM, et al. Is prostate cancer different in black men? Answers from 3 natural history models. Cancer 2017; 123:2312-2319.

2. US Preventive Services Task Force. Final Recommendation Statement: Prostate Cancer: Screening. Available at: https://http://www. uspreventiveservicestaskforce.org/Page/Document/RecommendationStatementFinal/prostate-cancer-screening. Accessed February 1, 2017.

3. US Preventive Services Task Force. Final Research Plan: Prostate Cancer: Screening. Available at: https://www.uspreventiveservicestaskforce.org/Page/Document/final-research-plan/prostate-cancer-screening1. Accessed February 1, 2017.

4. Brooks DD. Prostate cancer screening in black men-new questions, few answers. Cancer. 2013;119:2206-2208.

5. Bokhorst LP, Roobol MJ. Ethnicity and prostate cancer: the way to solve the screening problem [serial online]? BMC Med. 2015;13:179.

6. Shenoy D, Packianathan S, Chen AM, Vijayakumar S. Do AfricanAmerican men need separate prostate cancer screening guidelines [serial online]? BMC Urol. 2016;16:19.

7. American Urological Association. PSA Testing for the Pretreatment Staging and Posttreatment Management of Prostate Cancer: 2013 Revision of 2009 Best Practice Statement. Available at: https:// http://www.auanet.org/education/guidelines/prostate-specific-antigen. cfm. Accessed February 1, 2017.
8. National Comprehensive Cancer Network (NCCN). Prostate Cancer Early Detection. Fort Washington, PA: NCCN; 2014.

9. American Cancer Society. Prostate Cancer: Early Detection. Available at: http://www.cancer.org/cancer/prostatecancer/moreinformation/ prostatecancerearlydetection/prostate-cancer-early-detection-toc. Accessed February 1, 2017.

10. American Cancer Society. Cancer Facts \& Figures for African Americans 2016-2018. Available at: http://www.cancer.org/content/dam/ cancer-org/research/cancer-facts-and-statistics/cancer-facts-and-figuresfor-african-americans/cancer-facts-and-figures-for-african-americans2016-2018.pdf. Accessed January 23, 2017.

11. Powell IJ. Epidemiology and pathophysiology of prostate cancer in African-American men. J Urol. 2007;177:444-449.

12. Ahaghotu C, Tyler R, Sartor O. African American Participation in oncology clinical trials-focus on prostate cancer: implications, barriers, and potential solutions. Clin Genitourin Cancer. 2016;14: 105-116.

13. Andriole GL, Crawford ED, Grubb RL, et al. Mortality Results From a Randomized Prostate-Cancer Screening Trial. Available at: http://dx.doi.org/10.1056/NEJMoa0810696 360. Accessed January 23, 2017.

14. Schroder FH, Hugosson J, Roobol MJ, et al. Screening and Prostate-Cancer Mortality in a Randomized European Study. Available at: http://dx.doi.org/10.1056/NEJMoa0810084 360. Accessed January 23, 2017.

15. Drazer MW, Huo D, Eggener SE. National prostate cancer screening rates after the 2012 US Preventive Services Task Force recommendation discouraging prostate-specific antigen-based screening. J Clin Oncol. 2015;33:2416-2423.

16. Etzioni R, Gulati R. Recent trends in PSA testing and prostate cancer incidence: a look at context. JAMA Oncol. 2016;2:955-956.

17. Wallner LP, Hsu JW, Loo RK, Palmer-Toy DE, Schottinger JE, Jacobsen SJ. Trends in prostate-specific antigen screening, prostate biopsies, urology visits, and prostate cancer treatments from 2000 to 2012. Urology. 2015;86:498-505.

18. Jemal A, Ma J, Siegel R, Fedewa S, Brawley O, Ward EM. Prostate cancer incidence rates 2 years after the US Preventive Services Task Force recommendations against screening. JAMA Oncol. 2016;2: 1657-1660.

19. Banerji JS, Wolff EM, Massman JD 3rd, Odem-Davis K, Porter CR, Corman JM. Prostate needle biopsy outcomes in the era of the US Preventive Services Task Force recommendation against prostate specific antigen based screening. J Urol. 2016;195:6673.

20. Hutchinson R, Akhtar A, Haridas J, Bhat D, Roehrborn C, Lotan $Y$. Testing and referral patterns in the years surrounding the US Preventive Services Task Force recommendation against prostate-specific antigen screening. Cancer. 2016;122:3785-3793.

21. Gulati R, Tsodikov A, Etzioni R, et al. Expected population impacts of discontinued prostate-specific antigen screening. Cancer. 2014; 120:3519-3526.

22. Weiner AB, Matulewicz RS, Eggener SE, Schaeffer EM. Increasing incidence of metastatic prostate cancer in the United States (20042013). Prostate Cancer Prostatic Dis. 2016;19:395-397.

23. Hoffman RM, Meisner AL, Arap W, et al. Trends in United States prostate cancer incidence rates by age and stage, 1995-2012. Cancer Epidemiol Biomarkers Prev. 2016;25:259-263. 